\title{
Changes in Bovine Colostrum Metabolites during Early Postpartum Period Revealed by ${ }^{1} \mathrm{H}-\mathrm{NMR}$ Metabolomics Approach
}

\author{
S. Settachaimongkon ${ }^{\mathrm{a}, \mathrm{b}, \mathrm{c}, *}$, N. Wannakajeepiboon ${ }^{\mathrm{a}}$, P. Arunpunporn ${ }^{\mathrm{a}}$, W. Mekboonsonglarp ${ }^{\mathrm{d}}$, \& \\ D. Makarapong \\ a Department of Food Technology, Faculty of Science, Chulalongkorn University, Bangkok, 10330, Thailand \\ bOmics Sciences and Bioinformatics Center, Faculty of Science, Chulalongkorn University, Bangkok, 10330, Thailand \\ cEmerging Processes for Food Functionality Design Research Unit, Chulalongkorn University, Bangkok, 10330, Thailand \\ 'Scientific and Technological Research Equipment Centre (STREC), Chulalongkorn University, Bangkok, 10330, Thailand \\ ${ }^{\mathrm{e}}$ David Enterprise and Development Co., Ltd., Bangkok, 10240, Thailand \\ *Corresponding author: sarn.s@chula.ac.th \\ (Received 21-09-2020; Revised 23-11-2020; Accepted 17-12-2020)
}

\begin{abstract}
The objectives of this study were to characterize and compare non-volatile polar metabolite profiles of bovine colostrum, collected within $1 \mathrm{~h}$ and at $72 \mathrm{~h}$ after parturition, from crossbred Holstein cows raised in northeastern Thailand. The colostrum serum was characterized and compared using a nontargeted proton nuclear magnetic resonance ( $\left.{ }^{1} \mathrm{H}-\mathrm{NMR}\right)$ technique combined with chemometric analysis. Results demonstrated that the main effect of post-parturition time provided a significant impact on the physical properties and major chemical constituents of colostrum, while the influence of farm origin and sampling month were likely undetectable. The ${ }^{1} \mathrm{H}-\mathrm{NMR}$ technique enabled to identify 45 non-volatile polar metabolites in the samples. Partial least-squares-discriminant analysis (PLS-DA) allowed discrimination of colostrum metabolome not only according to different times after parturition, but also the origins of the farm as well as sampling months. Differential metabolites were statistically identified as potential biomarkers accountable for the discrimination. Besides basic nutritive compounds (amino acids and sugars), several bioactive metabolites such as ascorbate, creatine, carnitine, choline, acetylcarnitine, $\mathrm{N}$-acetylglucosamine, ornithine, orotate, and UPD-glucose could be successfully elucidated. Our finding reveals the application of non-targeted ${ }^{1} \mathrm{H}$-NMR metabolomics as an effective tool to assess the biomolecular profiles of bovine colostrum and their essential dynamics during the first three days after parturition.
\end{abstract}

Keywords: dairy; milk; colostrum; metabolomics; foodomics

\section{INTRODUCTION}

Bovine colostrum is a yellowish viscous fluid secreted by the mammary glands of dairy cattle immediately after parturition. The secretion of colostrum remains through the first three days or up to a maximum of one week post-partum and precedes the production of mature milk (McGrath et al., 2016). This initial milk confers highly digestible nutrients, growth factors, antimicrobial compounds, and immunologically active components, especially a collection of passively acquired antibodies called immunoglobulins (Ig), which are essential for the growth and development of newborns (Godhia \& Patel, 2013). These bioactive compounds are also important for the implication as functional ingredients in food and nutraceuticals industries (Puppel et al., 2019).

The composition of colostrum is considerably different from that of mature milk. It has been documented that bovine colostrum contains a lower amount of lactose and higher amounts of fats (polyunsaturated fatty acids, phospholipids, cholesterol), proteins (casein, whey proteins, Ig, lactoferrin, and lysozyme), oligosaccharides, vitamins, minerals, hormones, growth factors, cytokines, and nucleotides than mature milk (McGrath et al., 2016; Penchev Georgiev, 2008). Indeed, it should be mentioned that the compositions of colostrum relied on several factors, e.g., animal breeds, postpartum period, lactation cycle, length of the dry period of cows, animal physiology, animal nutrition, dairy production management, and environmental factors (McGrath et al., 2016; Puppel et al., 2019). However, the influence of these factors on the low molecular weight fraction, i.e., metabolite composition of bovine colostrum, is also interesting and requires further investigation.

Recently, metabolomics has been well recognized in dairy research (Goldansaz et al., 2017). Different mass spectrometry (MS)-based, e.g., LC/MS, GC/MS, and $\mathrm{CE} / \mathrm{MS}$, and nuclear magnetic resonance (NMR)-based analytical platforms have been extensively applied for comprehensive characterization of small molecular weight metabolites present in raw and commercial milk (Foroutan et al., 2019; Goldansaz et al., 2017; Scano et al., 2019) as well as cheese and yogurt products (Afshari et 
al., 2020; Settachaimongkon et al., 2017). Still, information regarding the metabolite profile of bovine colostrum and its changes prior to the transition to mature milk is limited (Goldansaz et al., 2017; Li et al., 2020; Qi et al., 2018; Zhao et al., 2018). Indeed, to our best knowledge, a metabolomics approach has never been applied for the characterization of colostrum from cows raised in tropical countries. This information is essential since the presence of these metabolites could be substantially associated with the bioactivity, nutritional property, and technological application of colostrum produced in the tropical region.

Therefore, the objectives of this study were to characterize and compare non-volatile polar metabolite profiles of bovine colostrum collected within $1 \mathrm{~h}$ (day-1) and at $72 \mathrm{~h}$ (day-3) postpartum from two selected dairy farms located in the northeastern part of Thailand. A total number of 32 individual cow colostrum samples were analyzed through a non-targeted ${ }^{1} \mathrm{H}-\mathrm{NMR}$ metabolomics approach. Finally, the ${ }^{1} \mathrm{H}-\mathrm{NMR}$-derived metabolomes of samples were compared by means of multivariate analysis.

\section{MATERIALS AND METHODS}

\section{Ethical Concern, Farm Selection, and Colostrum Sample Collection}

All animal-related protocols have been approved by Institutional Animal Care and Use Committee (BIO 00710/2562). The experiment was organized as a completely randomized design consisting of eight treatments ( 2 farms $\times 2$ sampling periods $\times 2$ days post-partum) with four biological replicates (individual cows). With respect to this, two medium-scale dairy farms (6590 cows) situated in the northeastern part of Thailand, i.e., Pak-Chong District, Nakhon-Ratchasima Province

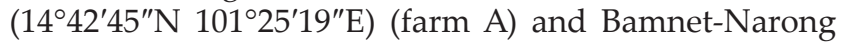
District, Chaiyaphum Province $\left(15^{\circ} 30^{\prime} 1^{\prime \prime} \mathrm{N} 101^{\circ} 41^{\prime} 11^{\prime \prime} \mathrm{E}\right)$ (farm B) were selected based on their most comparable farming management and milk quality records during the past six months. The distance between the two farms is $c a .120 \mathrm{~km}$. In each farm, colostrum from four crossbred Holstein dairy cows $(>80 \%$ Holstein) milking for the first lactation cycle, raised in either stall barn or free ranch, fed with roughages and concentrates (ca. 20\% proteins), were collected in two periods, i.e., October 2018 (period I; four cows) and January 2019 (period II; four cows). From each cow, $250 \mathrm{~mL}$ of colostrum was collected within $1 \mathrm{~h}$ (day-1) and at $72 \mathrm{~h}$ (day-3) after calving. Therefore, a total number of 32 colostrum samples were included. Samples were frozen and stored at $-35^{\circ} \mathrm{C}$ until the analysis (within 6 weeks).

\section{Determination of Physical Properties and Major Chemical Constituents of Colostrum}

The $\mathrm{pH}$ and titratable acidity of samples were determined according to ISO/IDF (2012) and expressed as $\%$ lactic acid (w/w). Viscosity was determined with an Alpha series rotational viscometer (V101003, Fungilab, Barcelona, Spain) using L model for the spindle with a shear rate set at $100 \mathrm{rpm}$ at $25^{\circ} \mathrm{C}$. Colostrum color was measured using a chromameter (CR-400/410, Konica Minolta Sensing, Singapore) and expressed as CIELAB $\left(L^{*}, a^{*}, b^{*}\right)$ color space coordinates; where $L^{*}$ was relative lightness from white to black, $a^{*}$ was relative value from red to green, and $b^{*}$ was relative value from yellow to blue (Gross et al., 2014). Major chemical constituents of colostrum, i.e., fat, protein, lactose, solid not fat $(\mathrm{SnF})$, and total solids (TS) contents were determined using a MilkoScan ${ }^{\mathrm{TM}}$ FT+ analyzer (FOSS, Hillerøed, Denmark).

\section{Sample Preparation and ${ }^{1} \mathrm{H}-\mathrm{NMR}$ Analysis}

The $\mathrm{pH}$ of the colostrum was adjusted to 6.0 using $1.0 \mathrm{~N} \mathrm{NaOH}$. Twenty-five $\mathrm{mL}$ of each sample was diluted 1:1 with milli-Q water (Picone et al., 2018). The ${ }^{1} \mathrm{H}-\mathrm{NMR}$ sample preparation was performed according to Luangwilai et al. (2021). In brief, lipids were removed by dichloromethane extraction, and then milk proteins were removed by ultra-centrifugation and ultrafiltration through a $3 \mathrm{kDa}$ molecular weight cutoff filter. The clear serum was mixed 1:1 (v/v) with phosphate buffer $\mathrm{pH} 6.0$ consisting of $1 \mathrm{mM}$ 3-(Trimethylsilyl) propionic-2, 2, 3, 3-d4 acid sodium salt (TSP) (Merck, Darmstadt, Germany) as internal standard. Finally, the mixture $(400 \mu \mathrm{L})$ was subjected to a $500 \mathrm{MHz}$ NOESYGPPR-1D- ${ }^{1} \mathrm{H}-\mathrm{NMR}$ spectrometer (Bruker, Rheinstetten, Germany) (Luangwilai et al., 2021).

\section{${ }^{1}$ H-NMR Spectra Processing and Data Acquisition}

${ }^{1} \mathrm{H}-\mathrm{NMR}$ spectra were corrected, pre-treated, and processed through binning (0.02 ppm interval) as described by Luangwilai et al. (2020). Metabolite identification was performed according to Chenomx NMR suite 8.2 library (Chenomx Inc., Canada), Livestock Metabolome Database (www.lmdb.ca), Milk Composition Database (www.mcdb.ca), and kinds of literature (Foroutan et al., 2019; Luangwilai et al., 2021; Picone et al., 2018; Scano et al., 2019; Zhao et al., 2018). The sum of signal intensity corresponding to respective metabolites was expressed in arbitrary units.

\section{Statistical Analysis}

ANOVA with multiple comparisons by Tukey's test was performed using IBM-SPSS 22.0 statistics package (SPSS Inc., Chicago, IL, USA). A probability at $\mathrm{p} \leq 0.05$ was considered statistically significant. ${ }^{1} \mathrm{H}-\mathrm{NMR}$ metabolomics data were normalized before subjecting to chemometric analysis (Settachaimongkon et al., 2014). Heat-map visualization combined with Pearson's correlation-based hierarchical clustering (HCA) and partial least-squares-discriminant analysis (PLS-DA) was performed using MetaboAnalyst 4.0 software (www.metaboanalyst.ca). The quality of the model was expressed by $R^{2}$ (accuracy) and $Q^{2}$ values (predictability) (Cui et al., 2019). Variable important in projection (VIP) scores with a value greater than 1.0 were used to screen out potential biomarker metabolites accountable for the discrimination (Cui et al., 2019). 


\section{RESULTS}

\section{Physical Properties and Major Chemical Constituents of Colostrum}

A significantly lower level of $\mathrm{pH}$ was observed in samples collected on day-1 compared to day-3 postpartum $(\mathrm{p}<0.05)$ (Table 1$)$. In association with $\mathrm{pH}$, a significantly higher titratable acidity was observed in samples collected on day-1 compared to day-3 $(\mathrm{p}<0.05)$ postpartum (Table 1). Significant influences of farm and sampling period on the $\mathrm{pH}$ and titratable acidity of colostrum were particularly observed within the group of samples collected on day-3 postpartum $(\mathrm{p}<0.05)$.

The viscosity values $(\mathrm{cP})$ of colostrum samples are presented in Table 1. Results demonstrated a significant reduction of viscosity in colostrum collected on day-3 compared to day-1 postpartum $(\mathrm{p}<0.05)$. A significant variation in viscosity was only observed among samples collected on day-3 postpartum $(\mathrm{p}<0.05)$. The influence of farm and sampling month was remarkably noticed. Samples collected from farm B exhibited a significantly higher viscosity compared to those from farm A. In addition, a significant difference between the two sampling months was only observed in samples collected from farm $B$.

For changes in colostrum color, no significant difference in the $L^{*}$ (lightness) and $a^{*}$ (red-green) values were observed among colostrum samples (Table 1). However, the positive $b^{*}$ values (yellowness) were significantly reduced in samples collected on day-3 compared to day-1 postpartum $(\mathrm{p}<0.05)$ (Figure 1). A significant influence of farm on the $b^{*}$ values was observed in samples collected on the same day postpartum $(p<0.05)$.

Regarding the variations in major chemical constituents, no significant difference in the fat content

Table 1. Physicochemical properties and major chemical compositions of bovine colostrum

\begin{tabular}{|c|c|c|c|c|c|c|c|c|}
\hline \multirow{3}{*}{$\begin{array}{l}\text { Colostrum } \\
\text { properties }\end{array}$} & \multicolumn{4}{|c|}{ Day $1(1 \mathrm{~h})$ after parturition } & \multicolumn{4}{|c|}{ Day $3(72 \mathrm{~h})$ after parturition } \\
\hline & \multicolumn{2}{|c|}{ Farm A } & \multicolumn{2}{|c|}{ Farm B } & \multicolumn{2}{|c|}{ Farm A } & \multicolumn{2}{|c|}{ Farm B } \\
\hline & Period I* & Period II ${ }^{* *}$ & Period I & Period II & Period I & Period II & Period I & Period II \\
\hline $\mathrm{pH}$ & $6.06 \pm 0.09^{d}$ & $6.09 \pm 0.06^{\mathrm{d}}$ & $6.03 \pm 0.07^{\mathrm{d}}$ & $6.02 \pm 0.07^{\mathrm{d}}$ & $6.35 \pm 0.06^{\mathrm{ab}}$ & $6.43 \pm 0.06^{\mathrm{a}}$ & $6.23 \pm 0.13^{\mathrm{bc}}$ & $6.14 \pm 0.05^{\mathrm{cd}}$ \\
\hline $\begin{array}{l}\text { Titratable acidity } \\
(\% \text { LA) }\end{array}$ & $0.40 \pm 0.04^{\mathrm{a}}$ & $0.40 \pm 0.04^{\mathrm{a}}$ & $0.42 \pm 0.04^{\mathrm{a}}$ & $0.40 \pm 0.02^{\mathrm{a}}$ & $0.18 \pm 0.02^{c}$ & $0.18 \pm 0.03^{c}$ & $0.29 \pm 0.08^{b}$ & $0.35 \pm 0.05^{\mathrm{ab}}$ \\
\hline Viscosity $(\mathrm{cP})$ & $139.98 \pm 54.17^{\mathrm{ab}}$ & $87.23 \pm 55.11^{\mathrm{ab}}$ & $124.19 \pm 39.75^{\mathrm{ab}}$ & $216.11 \pm 27.78^{\mathrm{a}}$ & $17.81 \pm 3.85^{c}$ & $20.63 \pm 14.63^{c}$ & $89.18 \pm 5.5^{b}$ & $165.54 \pm 49.25^{\mathrm{a}}$ \\
\hline \multicolumn{9}{|l|}{ CIE coordinates: } \\
\hline Brightness $\left(L^{*}\right)$ & $60.60 \pm 15.00$ & $71.19 \pm 13.69$ & $70.65 \pm 4.94$ & $71.98 \pm 2.88$ & $74.21 \pm 4.82$ & $74.30 \pm 7.69$ & $72.56 \pm 6.26$ & $75.30 \pm 2.07$ \\
\hline Green-red $\left(a^{*}\right)$ & $-2.22 \pm 0.87$ & $-3.60 \pm 0.49$ & $-2.28 \pm 0.87$ & $-1.62 \pm 0.34$ & $-3.22 \pm 0.78$ & $-2.83 \pm 0.55$ & $-2.31 \pm 0.57$ & $-2.54 \pm 0.48$ \\
\hline Blue-yellow $\left(b^{*}\right)$ & $15.32 \pm 4.59^{\mathrm{b}}$ & $14.27 \pm 2.70^{\mathrm{b}}$ & $25.06 \pm 3.41^{\mathrm{a}}$ & $24.93 \pm 5.48^{\mathrm{a}}$ & $5.50 \pm 1.66^{c}$ & $6.14 \pm 2.67 \mathrm{c}$ & $15.04 \pm 3.02^{\mathrm{b}}$ & $19.71 \pm 4.81^{\mathrm{ab}}$ \\
\hline Fat $(\%)$ & $5.91 \pm 1.19^{\mathrm{ab}}$ & $6.17 \pm 2.05^{\mathrm{ab}}$ & $5.28 \pm 1.27^{\mathrm{ab}}$ & $9.34 \pm 4.61^{\mathrm{a}}$ & $3.09 \pm 0.77 \mathrm{~b}$ & $7.38 \pm 5.70^{\mathrm{ab}}$ & $8.60 \pm 4.29^{a}$ & $5.78 \pm 2.39^{\mathrm{ab}}$ \\
\hline Protein (\%) & $22.40 \pm 3.08^{\mathrm{a}}$ & $17.69 \pm 4.06^{\mathrm{ab}}$ & $18.86 \pm 2.90^{\mathrm{ab}}$ & $17.80 \pm 4.76^{\mathrm{ab}}$ & $5.52 \pm 1.08^{\mathrm{d}}$ & $6.37 \pm 1.16^{\mathrm{d}}$ & $9.45 \pm 6.42^{\mathrm{cd}}$ & $13.77 \pm 8.20^{\mathrm{bc}}$ \\
\hline Lactose (\%) & $2.21 \pm 0.57^{\mathrm{bc}}$ & $2.86 \pm 0.40^{\mathrm{bc}}$ & $2.68 \pm 0.25^{\mathrm{bc}}$ & $2.04 \pm 0.63^{c}$ & $4.61 \pm 0.32^{\mathrm{a}}$ & $4.50 \pm 1.30^{\mathrm{a}}$ & $3.21 \pm 0.68^{\mathrm{b}}$ & $3.01 \pm 0.73^{\mathrm{bc}}$ \\
\hline $\operatorname{SnF}(\%)$ & $30.42 \pm 2.88^{\mathrm{a}}$ & $26.60 \pm 4.73^{\mathrm{ab}}$ & $26.42 \pm 4.19^{\mathrm{ab}}$ & $29.96 \pm 3.19^{a}$ & $12.90 \pm 1.58^{\mathrm{c}}$ & $19.24 \pm 9.24^{\text {bc }}$ & $21.73 \pm 8.22^{\mathrm{abc}}$ & $22.34 \pm 10.00^{\mathrm{abc}}$ \\
\hline TS (\%) & $25.61 \pm 2.59^{\mathrm{a}}$ & $21.45 \pm 3.75^{\mathrm{ab}}$ & $22.32 \pm 2.96^{\mathrm{ab}}$ & $21.30 \pm 4.59^{\mathrm{ab}}$ & $10.78 \pm 0.70^{c}$ & $12.32 \pm 2.76^{c}$ & $13.80 \pm 5.98^{c}$ & $17.63 \pm 7.74^{\mathrm{bc}}$ \\
\hline
\end{tabular}

Note: Values are the average from four individual cow samples \pm SD corresponding to the respective day after parturition, farm, and sampling period $\mathrm{SnF}=$ solids not fat; TS $=$ total solids; ${ }^{*}=$ Period I corresponds to sampling during October $2018 ;{ }^{* *}=$ Period II corresponds to sampling during January 2019. Means in the same row with different superscripts differ significantly $(\mathrm{p} \leq 0.05)$.

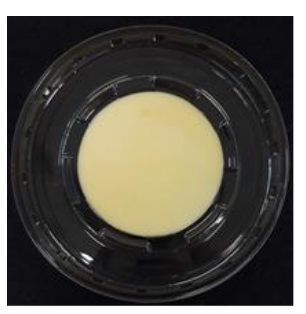

Farm A-Period I-Day-1

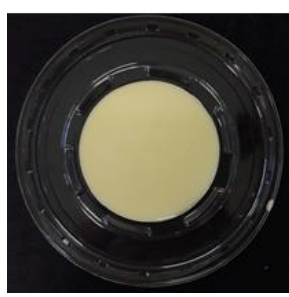

Farm A-Period II-Day-1

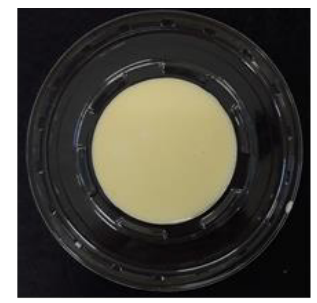

Farm B-Period I-Day-1

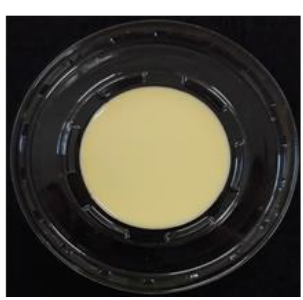

Farm B-Period II-Day-1

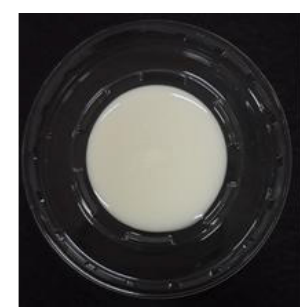

Farm A-Period I-Day-3

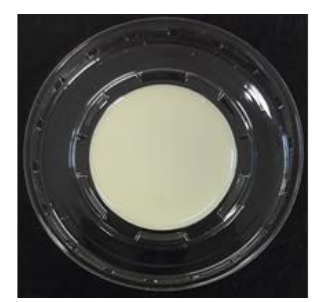

Farm A-Period II-Day-3

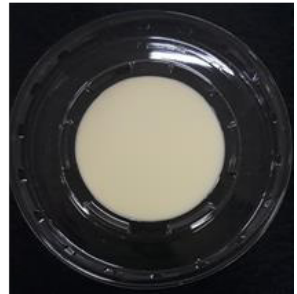

Farm B-Period I-Day-3

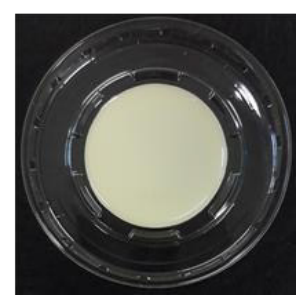

Farm B-Period II-Day-3

Figure 1. Spectrum of colostrum color in representative samples corresponding to the respective farm, sampling period, and day after parturition. 
was observed among colostrum samples (Table 1). However, it was remarkable that the concentration of proteins significantly decreased while the concentration of lactose significantly increased in samples collected on day-3 compared to those of day-1 postpartum $(p<0.05)$. Fluctuations in these two major components provided a significant influence on the SnF and TS content of the samples $(\mathrm{p}<0.05)$. Also, a significant influence of farm on protein, lactose, $\mathrm{SnF}$, and TS contents was noticed among samples collected on the same day postpartum $(\mathrm{p}<0.05)$.

\section{Identification of Colostrum Metabolites and Chemometric Analysis}

A total of 45 non-volatile polar metabolites were identified in colostrum samples of this study (Figure 2; Table 2-3). The quantitative relationships of ${ }^{1} \mathrm{H}-\mathrm{NMR}$ derived metabolomic profiles among samples $(n=32)$ are shown in Figure 2. The Pearson's correlation-based dendrogram revealed that colostrum samples collected on day-1 (cluster A) and day-3 (cluster B) postpartum could be grouped into different clusters according to their ${ }^{1} \mathrm{H}-\mathrm{NMR}$ derived metabolomic profiles. Results demonstrated that the majority of metabolites, especially vari-

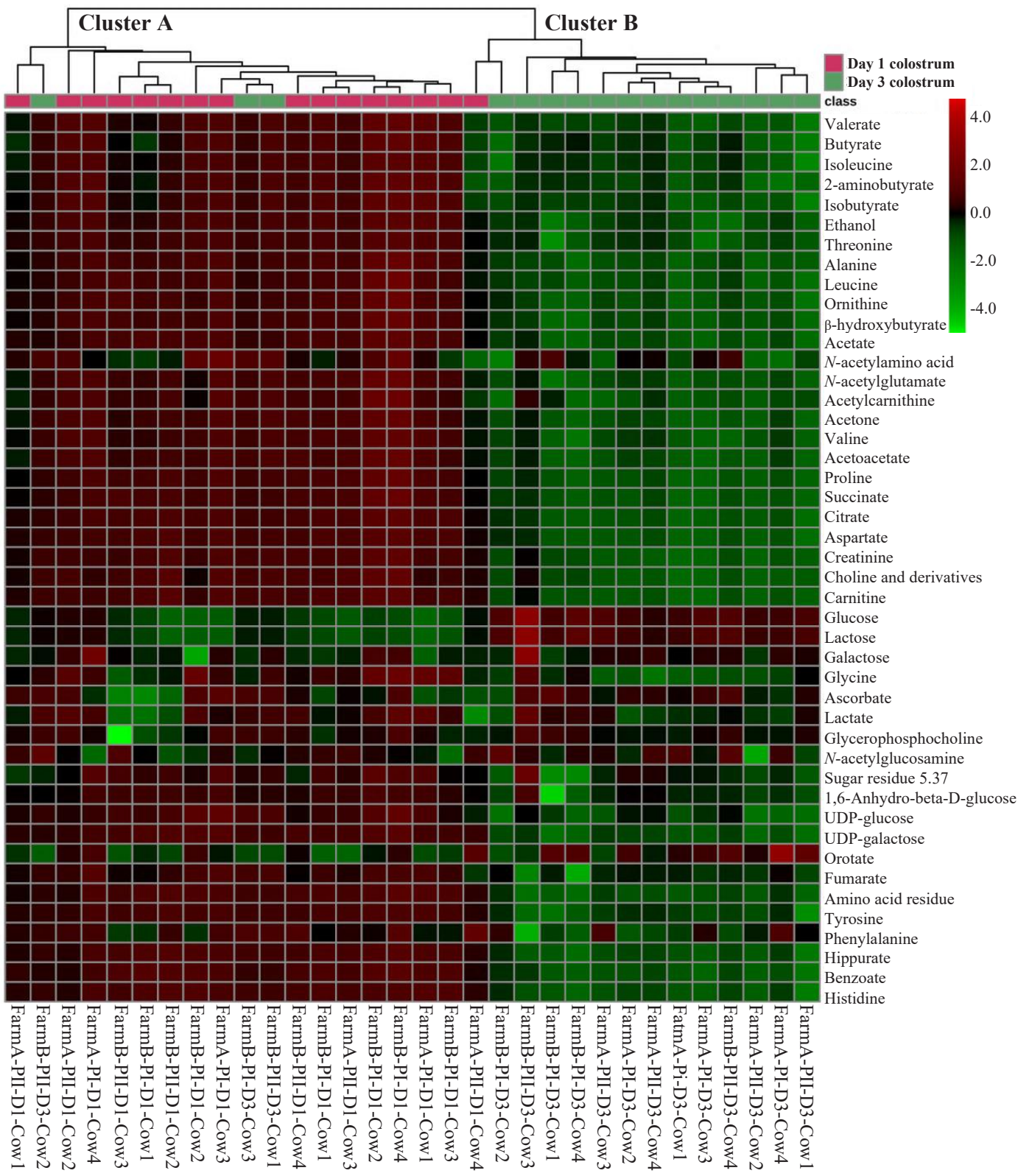

Figure 2. Heat-map visualization and hierarchical clustering of non-volatile polar metabolite profiles of colostrum collected from individual cows corresponding to the respective farm $(\mathrm{F})$, sampling month (period; $\mathrm{P}$ ), and time after parturition (day; D). Dendrogram represents sample clusters based on Pearson's correlation coefficient with average linkage. Each square in the heat-map expresses normalized non-volatile polar metabolite content respected to the color range. The red color indicates higher content of the corresponding compound. For interpretation of the references to color in this figure, the reader is referred to the web version of this article or the relative quantification of metabolites in supplementary Table S1. 
ables corresponding to amino acids and organic acids, were present in high relative abundance (shown in red) in colostrum samples collected on day-1 postpartum (cluster A). On the other hand, variables in the sugar region were present in high relative abundance in the samples collected on day-3 postpartum (cluster B). The integrated intensities of all metabolites were expressed in $\log _{10}$ transformed (arbitrary unit) and statistically compared using ANOVA (Table 3).

A supervised pattern recognition PLS-DA was performed to discriminate the ${ }^{1} \mathrm{H}-\mathrm{NMR}$ derived metabolomic profiles among colostrum samples $(n=32)$.

Table 2. Assignment table of the non-volatile polar metabolites present in the ${ }^{1} \mathrm{H}-\mathrm{NMR}$ spectra of bovine colostrum

\begin{tabular}{|c|c|c|}
\hline Chemical group & Compound name & ${ }^{1} \mathrm{H}$ chemical shift, $\mathrm{ppm}^{\mathrm{a}}$ \\
\hline Alcohol & Ethanol & $3.65(\mathrm{~m})^{\mathrm{b}}, 1.17(\mathrm{t})$ \\
\hline \multirow[t]{17}{*}{ Amino acid and derivatives } & Alanine & $3.81(\mathrm{~m}), 1.47(\mathrm{~d})$ \\
\hline & Amino acid residues & $8.23(\mathrm{~m}), 8.11(\mathrm{~m}), 7.29(\mathrm{~m}), 6.85(\mathrm{~m})$ \\
\hline & 2-aminobutyrate & $3.70(\mathrm{t}), 1.88(\mathrm{~m}), 0.96(\mathrm{t})$ \\
\hline & Aspartate & $3.91(\mathrm{~s}), 2.80(\mathrm{~m}), 2.67(\mathrm{~m})$ \\
\hline & Creatine and Creatinine & $4.07(\mathrm{~s}), 3.95(\mathrm{~s}), 3.05(\mathrm{~s}), 3.03(\mathrm{~s})$ \\
\hline & Glycine & $3.55(\mathrm{~s})$ \\
\hline & Histidine & $8.41(\mathrm{~s}), 3.31(\mathrm{~m}), 3.29(\mathrm{~m})$ \\
\hline & Isoleucine & $3.69(\mathrm{~d}), 0.99(\mathrm{~d}), 0.93(\mathrm{t})$ \\
\hline & Leucine & $3.75(\mathrm{~m}), 1.71(\mathrm{~m}), 0.97(\mathrm{t})$ \\
\hline & $\mathrm{N}$-acetylamino acid & $2.05(\mathrm{~s}), 2.01(\mathrm{~s})$ \\
\hline & $\mathrm{N}$-acetylglutamate & $4.10(\mathrm{~m}), 2.22(\mathrm{t}), 2.02(\mathrm{~s}), 1.86(\mathrm{~m})$ \\
\hline & Ornithine & $3.78(\mathrm{t}), 3.05(\mathrm{t}), 1.93) \mathrm{m}), 1.82(\mathrm{~m}), 1.73(\mathrm{~m})$ \\
\hline & Phenylalanine & $7.40(\mathrm{~m}), 7.32(\mathrm{~d}), 3.99(\mathrm{~m}), 3.28(\mathrm{~m}), 3.12(\mathrm{~m})$ \\
\hline & Proline & $3.43(\mathrm{~m}), 3.35(\mathrm{~m}), 2.35(\mathrm{~m}), 2.03(\mathrm{~m})$ \\
\hline & Tyrosine & $6.91(\mathrm{~d}), 3.97(\mathrm{~m}), 3.20(\mathrm{~m}), 3.03(\mathrm{~m})$ \\
\hline & Threonine & $4.29(\mathrm{~m}), 3.85(\mathrm{~d}), 1.33(\mathrm{~d})$ \\
\hline & Valine & $3.63(\mathrm{~d}), 2.25(\mathrm{~m}), 1.05(\mathrm{~d}), 0.99(\mathrm{~d})$ \\
\hline \multirow[t]{8}{*}{ Carbohydrate and derivatives } & 1,6-Anhydro- $\beta$-D-glucose & $5.47(\mathrm{~s}), 4.07(\mathrm{~d}), 3.74(\mathrm{~m}), 3.52(\mathrm{~s})$ \\
\hline & Galactose & $5.29(\mathrm{~d}), 4.59(\mathrm{~d}), 4.09(\mathrm{~m}), 3.81(\mathrm{~m}), 3.51(\mathrm{~m})$ \\
\hline & Glucose & $5.25(\mathrm{~m}), 4.65(\mathrm{~d}), 3.85(\mathrm{~m}), 3.41(\mathrm{~m})$ \\
\hline & Lactose & $5.23(\mathrm{~d}), 4.69(\mathrm{~d}), 4.47(\mathrm{~d}), 3.97(\mathrm{~m}), 3.71(\mathrm{~m}), 3.31(\mathrm{~m})$ \\
\hline & N-Acetylglucosamine & $8.03(\mathrm{~d}), 5.53(\mathrm{~d}), 5.21(\mathrm{~d}), 2.09(\mathrm{~s})$ \\
\hline & UDP-galactose & $5.99(\mathrm{~m}), 5.67(\mathrm{~m})$ \\
\hline & UDP-glucose & $5.61(\mathrm{~d}), 4.15(\mathrm{~m})$ \\
\hline & Sugar residues & $5.45(\mathrm{~m})$ \\
\hline Carbonyl compound & Acetone & $2.21(\mathrm{~s})$ \\
\hline \multirow[t]{14}{*}{ Organic acid } & Acetate & $1.93(\mathrm{~s})$ \\
\hline & Acetoacetate & $2.27(\mathrm{~s})$ \\
\hline & Ascorbate & $4.51(\mathrm{~d}), 3.75(\mathrm{~m})$ \\
\hline & Benzoate & $7.89(\mathrm{~d}), 7.55(\mathrm{~d})$ \\
\hline & Butyrate & $2.17(\mathrm{t}), 1.55(\mathrm{~m}), 0.89(\mathrm{t})$ \\
\hline & Citrate & $2.71(\mathrm{~d}), 2.55(\mathrm{~d})$ \\
\hline & Fumarate & $6.53(\mathrm{~s})$ \\
\hline & Hippurate & $8.52(\mathrm{~s}), 7.83(\mathrm{~d}), 7.59(\mathrm{~m})$ \\
\hline & $\beta$-Hydroxybutyrate & $0.89(\mathrm{t})$ \\
\hline & Isobutyrate & $2.35(\mathrm{~m}), 1.05(\mathrm{~d})$ \\
\hline & Lactate & $4.13(\mathrm{~m}) 1.35(\mathrm{~d})$ \\
\hline & Orotate & $6.19(\mathrm{~s})$ \\
\hline & Succinate & $2.43(\mathrm{~s})$ \\
\hline & Valerate and derivatives & $3.85(\mathrm{~m}), 2.37(\mathrm{~s}), 2.03(\mathrm{~m}), 1.29(\mathrm{~m}), 0.85(\mathrm{t})$ \\
\hline \multirow[t]{4}{*}{ Lipid derivatives } & Acetyl-carnitine & $5.61(\mathrm{~m}), 3.17(\mathrm{~s}), 2.15(\mathrm{~s})$ \\
\hline & Carnitine & $3.23(\mathrm{~s}), 3.43(\mathrm{~d}), 3.47(\mathrm{~d})$ \\
\hline & Choline and derivatives & $3.23(\mathrm{~s}), 3.19(\mathrm{~s})$ \\
\hline & Glycerophosphocholine & $4.31(\mathrm{~m}), 3.61(\mathrm{~m}), 3.23(\mathrm{~s})$ \\
\hline
\end{tabular}

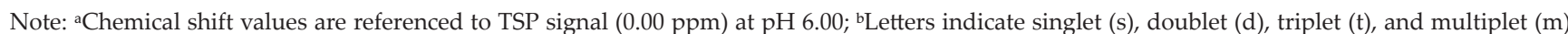
multiplicity of $1 \mathrm{H}-\mathrm{NMR}$ peaks, respectively. 
Table 3. Presumptive non-volatile polar metabolites identified in bovine colostrum using a high resolution NOESY-1D-1H-NMR spectroscopy $(500 \mathrm{MHz})$

\begin{tabular}{|c|c|c|c|c|c|c|c|c|c|}
\hline \multirow{3}{*}{$\begin{array}{l}\text { Chemical } \\
\text { group }\end{array}$} & \multirow{3}{*}{ Compound } & \multicolumn{4}{|c|}{ Day 1 after parturition } & \multicolumn{4}{|c|}{ Day 3 after parturition } \\
\hline & & \multirow{2}{*}{$\begin{array}{l}\text { Farm A } \\
\text { Period I* }\end{array}$} & \multicolumn{3}{|c|}{ Farm B } & \multirow{2}{*}{$\begin{array}{l}\text { Farm A } \\
\text { Period I }\end{array}$} & \multicolumn{3}{|c|}{ Farm B } \\
\hline & & & Period II** & Period I & Period II & & Period II & Period I & Period II \\
\hline Alcohol & Ethanol & $6.89 \pm 0.09^{\mathrm{a}}$ & $6.54 \pm 0.27^{\mathrm{ab}}$ & $6.89 \pm 0.27^{\mathrm{a}}$ & $6.71 \pm 0.13^{\mathrm{ab}}$ & $5.72 \pm 0.37^{\mathrm{c}}$ & $5.72 \pm 0.26^{c}$ & $5.71 \pm 0.77^{\mathrm{c}}$ & $6.13 \pm 0.78^{\text {bc }}$ \\
\hline \multirow{17}{*}{$\begin{array}{l}\text { Amino } \\
\text { acids and } \\
\text { derivatives }\end{array}$} & Alanine & $6.90 \pm 0.05^{\mathrm{a}}$ & $6.65 \pm 0.17^{\mathrm{ab}}$ & $6.97 \pm 0.20^{\mathrm{a}}$ & $6.81 \pm 0.03^{\mathrm{a}}$ & $6.01 \pm 0.11^{\mathrm{d}}$ & $6.00 \pm 0.14^{\mathrm{d}}$ & $6.19 \pm 0.41^{\mathrm{cd}}$ & $6.46 \pm 0.31^{\text {bc }}$ \\
\hline & Amino acid residue & $8.64 \pm 0.02^{\mathrm{a}}$ & $8.47 \pm 0.08^{\mathrm{ab}}$ & $8.67 \pm 0.05^{\mathrm{a}}$ & $8.61 \pm 0.05^{\mathrm{a}}$ & $8.04 \pm 0.11^{c}$ & $7.98 \pm 0.13^{c}$ & $8.11 \pm 0.36^{c}$ & $8.21 \pm 0.38^{\mathrm{bc}}$ \\
\hline & 2-aminobutyrate & $6.25 \pm 0.13^{\mathrm{a}}$ & $5.65 \pm 0.63^{\mathrm{ab}}$ & $6.17 \pm 0.49^{a}$ & $5.96 \pm 0.22^{\mathrm{ab}}$ & $4.77 \pm 0.39^{c}$ & $4.86 \pm 0.44^{\mathrm{c}}$ & $5.31 \pm 0.48^{\mathrm{bc}}$ & $5.67 \pm 0.44^{\mathrm{ab}}$ \\
\hline & Aspartate & $6.73 \pm 0.05^{\mathrm{a}}$ & $6.58 \pm 0.14^{\mathrm{ab}}$ & $6.83 \pm 0.09^{\mathrm{a}}$ & $6.74 \pm 0.07^{\mathrm{a}}$ & $5.80 \pm 0.11^{\mathrm{d}}$ & $5.77 \pm 0.18^{\mathrm{d}}$ & $6.13 \pm 0.40^{c}$ & $6.36 \pm 0.34^{\mathrm{bc}}$ \\
\hline & Creatinine and Creatine & $7.07 \pm 0.08^{\mathrm{ab}}$ & $6.98 \pm 0.14^{\mathrm{ab}}$ & $7.21 \pm 0.13^{\mathrm{a}}$ & $7.11 \pm 0.08^{\mathrm{ab}}$ & $6.24 \pm 0.12^{\mathrm{d}}$ & $6.23 \pm 0.05^{\mathrm{d}}$ & $6.60 \pm 0.32^{c}$ & $6.84 \pm 0.31^{\mathrm{bc}}$ \\
\hline & Glycine & $6.14 \pm 0.30^{\mathrm{a}}$ & $5.76 \pm 0.32^{\mathrm{ab}}$ & $6.12 \pm 0.49^{a}$ & $5.51 \pm 0.42^{\mathrm{bc}}$ & $5.05 \pm 0.13^{c}$ & $5.03 \pm 0.44^{\mathrm{c}}$ & $5.46 \pm 0.18^{\mathrm{bc}}$ & $5.75 \pm 0.46^{\mathrm{ab}}$ \\
\hline & Histidine & $7.52 \pm 0.05^{\mathrm{a}}$ & $7.28 \pm 0.11^{\mathrm{ab}}$ & $7.52 \pm 0.03^{\mathrm{a}}$ & $7.47 \pm 0.07^{\mathrm{a}}$ & $6.60 \pm 0.16^{\mathrm{cd}}$ & $6.39 \pm 0.32^{\mathrm{d}}$ & $6.77 \pm 0.48^{\mathrm{cd}}$ & $6.99 \pm 0.41^{\mathrm{bc}}$ \\
\hline & Isoleucine & $6.87 \pm 0.12^{\mathrm{a}}$ & $6.24 \pm 0.58^{\mathrm{ab}}$ & $6.80 \pm 0.44^{\mathrm{a}}$ & $6.55 \pm 0.24^{\mathrm{a}}$ & $5.46 \pm 0.29^{\mathrm{bc}}$ & $5.23 \pm 0.80^{c}$ & $5.69 \pm 0.87^{\mathrm{bc}}$ & $6.29 \pm 0.43^{\mathrm{ab}}$ \\
\hline & Leucine & $7.41 \pm 0.0^{\mathrm{ab}}$ & $7.21 \pm 0.17^{\mathrm{bc}}$ & $7.54 \pm 0.22^{\mathrm{a}}$ & $7.37 \pm 0.05^{\mathrm{ab}}$ & $6.62 \pm 0.10^{\mathrm{e}}$ & $6.58 \pm 0.23^{e}$ & $6.85 \pm 0.29^{\mathrm{de}}$ & $7.03 \pm 0.26^{\mathrm{cd}}$ \\
\hline & $\mathrm{N}$-acetylamino acid & $6.96 \pm 0.23^{\mathrm{a}}$ & $6.71 \pm 0.30^{\mathrm{ab}}$ & $6.82 \pm 0.32^{\mathrm{ab}}$ & $6.67 \pm 0.08^{\mathrm{ab}}$ & $6.54 \pm 0.28^{\mathrm{ab}}$ & $6.46 \pm 0.22^{\mathrm{b}}$ & $6.66 \pm 0.47^{\mathrm{ab}}$ & $6.93 \pm 0.09^{\mathrm{a}}$ \\
\hline & N-acetyl glutamate & $6.30 \pm 0.18^{\mathrm{ab}}$ & $6.11 \pm 0.28^{\mathrm{ab}}$ & $6.45 \pm 0.26^{\mathrm{a}}$ & $6.27 \pm 0.05^{\mathrm{ab}}$ & $5.53 \pm 0.15^{c}$ & $5.49 \pm 0.16^{c}$ & $5.52 \pm 0.52^{\mathrm{c}}$ & $5.99 \pm 0.37^{\mathrm{b}}$ \\
\hline & Ornithine & $7.07 \pm 0.08^{\mathrm{a}}$ & $6.91 \pm 0.17^{\mathrm{ab}}$ & $7.22 \pm 0.21^{\mathrm{a}}$ & $7.07 \pm 0.04^{\mathrm{a}}$ & $6.23 \pm 0.10^{\mathrm{d}}$ & $6.24 \pm 0.27^{\mathrm{d}}$ & $6.41 \pm 0.40^{\mathrm{cd}}$ & $6.68 \pm 0.28^{\mathrm{bc}}$ \\
\hline & Phenylalanine & $7.55 \pm 0.10$ & $7.62 \pm 0.09$ & $7.54 \pm 0.11$ & $7.55 \pm 0.11$ & $7.49 \pm 0.15$ & $7.51 \pm 0.11$ & $7.49 \pm 0.17$ & $7.37 \pm 0.35$ \\
\hline & Proline & $7.18 \pm 0.06^{\mathrm{a}}$ & $6.98 \pm 0.18^{\mathrm{ab}}$ & $7.28 \pm 0.18^{\mathrm{a}}$ & $7.14 \pm 0.03^{\mathrm{a}}$ & $6.31 \pm 0.14^{\mathrm{d}}$ & $6.33 \pm 0.13^{\mathrm{d}}$ & $6.55 \pm 0.37^{\mathrm{cd}}$ & $6.81 \pm 0.33^{\text {bc }}$ \\
\hline & Threonine & $6.46 \pm 0.07^{\mathrm{a}}$ & $6.14 \pm 0.25^{\mathrm{a}}$ & $6.49 \pm 0.23^{\mathrm{a}}$ & $6.32 \pm 0.11^{\mathrm{a}}$ & $5.07 \pm 0.53^{\mathrm{b}}$ & $5.26 \pm 0.29^{\mathrm{b}}$ & $5.07 \pm 1.13^{\mathrm{b}}$ & $5.76 \pm 0.69^{\mathrm{ab}}$ \\
\hline & Tyrosine & $7.57 \pm 0.03^{\mathrm{a}}$ & $7.33 \pm 0.13^{\mathrm{abc}}$ & $7.59 \pm 0.06^{\mathrm{a}}$ & $7.53 \pm 0.07^{\mathrm{ab}}$ & $6.64 \pm 0.19^{\mathrm{cd}}$ & $6.23 \pm 0.75^{\mathrm{d}}$ & $6.55 \pm 0.74^{\mathrm{d}}$ & $6.83 \pm 0.72^{\mathrm{bcd}}$ \\
\hline & Valine & $6.66 \pm 0.09^{\mathrm{a}}$ & $6.37 \pm 0.29^{\mathrm{a}}$ & $6.70 \pm 0.26^{\mathrm{a}}$ & $6.51 \pm 0.11^{\mathrm{a}}$ & $5.66 \pm 0.26^{\mathrm{b}}$ & $5.66 \pm 0.22^{\mathrm{b}}$ & $5.69 \pm 0.63^{\mathrm{b}}$ & $6.13 \pm 0.58^{\mathrm{ab}}$ \\
\hline \multirow{9}{*}{$\begin{array}{l}\text { Carbohydrates } \\
\text { and } \\
\text { derivatives }\end{array}$} & $\begin{array}{l}\text { 1,6-Anhydro- } \beta \text {-D- } \\
\text { glucose }\end{array}$ & $7.30 \pm 0.06^{\mathrm{a}}$ & $7.05 \pm 0.11^{\mathrm{a}}$ & $7.28 \pm 0.11^{\mathrm{a}}$ & $7.23 \pm 0.10^{\mathrm{a}}$ & $6.85 \pm 0.09^{\mathrm{a}}$ & $6.71 \pm 0.23^{\mathrm{ab}}$ & $6.18 \pm 1.02^{\mathrm{b}}$ & $7.07 \pm 0.24^{a}$ \\
\hline & Ascorbate & $7.02 \pm 0.59^{\mathrm{ab}}$ & $6.98 \pm 0.44^{\mathrm{ab}}$ & $6.58 \pm 0.83^{\mathrm{ab}}$ & $6.28 \pm 0.64^{b}$ & $7.02 \pm 0.24^{\mathrm{ab}}$ & $6.97 \pm 0.18^{\mathrm{ab}}$ & $7.14 \pm 0.50^{\mathrm{a}}$ & $7.39 \pm 0.03^{\mathrm{a}}$ \\
\hline & Galactose & $7.84 \pm 0.60$ & $8.00 \pm 0.10$ & $8.09 \pm 0.15$ & $7.96 \pm 0.07$ & $8.09 \pm 0.05$ & $8.05 \pm 0.11$ & $7.92 \pm 0.06$ & $8.24 \pm 0.33$ \\
\hline & Lactose & $9.19 \pm 0.14^{\mathrm{c}}$ & $9.29 \pm 0.10^{\mathrm{bc}}$ & $9.21 \pm 0.03^{c}$ & $9.21 \pm 0.08^{c}$ & $9.45 \pm 0.03^{\mathrm{ab}}$ & $9.45 \pm 0.04^{\mathrm{ab}}$ & $9.45 \pm 0.10^{\mathrm{ab}}$ & $9.50 \pm 0.22^{\mathrm{a}}$ \\
\hline & Glucose & $9.18 \pm 0.14^{\mathrm{c}}$ & $9.29 \pm 0.10^{\mathrm{bc}}$ & $9.21 \pm 0.03^{c}$ & $9.21 \pm 0.08^{c}$ & $9.45 \pm 0.03^{\mathrm{ab}}$ & $9.45 \pm 0.04^{\mathrm{ab}}$ & $9.45 \pm 0.10^{\mathrm{ab}}$ & $9.50 \pm 0.22^{\mathrm{a}}$ \\
\hline & $\mathrm{N}$-acetylglucosamine & $10.34 \pm 0.04$ & $10.38 \pm 0.01$ & $10.34 \pm 0.05$ & $10.37 \pm 0.04$ & $10.37 \pm 0.03$ & $10.32 \pm 0.10$ & $10.37 \pm 0.04$ & $10.40 \pm 0.03$ \\
\hline & Sugar residue & $7.91 \pm 0.13^{\mathrm{a}}$ & $7.64 \pm 0.11^{\mathrm{ab}}$ & $7.86 \pm 0.16^{\mathrm{ab}}$ & $7.80 \pm 0.16^{\mathrm{ab}}$ & $7.63 \pm 0.08^{\mathrm{ab}}$ & $7.49 \pm 0.20^{\mathrm{b}}$ & $7.14 \pm 0.46^{c}$ & $7.77 \pm 0.29^{\mathrm{ab}}$ \\
\hline & UDP-galactose & $8.16 \pm 0.04^{\mathrm{a}}$ & $8.07 \pm 0.04^{\mathrm{ab}}$ & $8.18 \pm 0.06^{\mathrm{a}}$ & $8.14 \pm 0.04^{\mathrm{a}}$ & $7.66 \pm 0.06^{\mathrm{c}}$ & $7.60 \pm 0.17^{c}$ & $7.68 \pm 0.31^{\mathrm{c}}$ & $7.92 \pm 0.17^{b}$ \\
\hline & UDP-glucose & $7.85 \pm 0.11^{\mathrm{a}}$ & $7.62 \pm 0.09^{\mathrm{ab}}$ & $7.80 \pm 0.16^{\mathrm{a}}$ & $7.72 \pm 0.07^{\mathrm{a}}$ & $7.35 \pm 0.18^{\text {bc }}$ & $7.21 \pm 0.25^{c}$ & $7.35 \pm 0.35^{\mathrm{bc}}$ & $7.66 \pm 0.12^{\mathrm{a}}$ \\
\hline $\begin{array}{l}\text { Carbonyl } \\
\text { compounds }\end{array}$ & Acetone & $6.41 \pm 0.09^{\mathrm{ab}}$ & $6.18 \pm 0.26^{\mathrm{ab}}$ & $6.49 \pm 0.24^{\mathrm{a}}$ & $6.32 \pm 0.08^{\mathrm{ab}}$ & $5.56 \pm 0.20^{\mathrm{d}}$ & $5.50 \pm 0.16^{\mathrm{d}}$ & $5.71 \pm 0.44^{\text {cd }}$ & $6.06 \pm 0.39^{\mathrm{bc}}$ \\
\hline \multirow{4}{*}{$\begin{array}{l}\text { Lipid } \\
\text { derivatives }\end{array}$} & Acetyl carnitine & $6.40 \pm 0.13^{\mathrm{a}}$ & $6.24 \pm 0.21^{\mathrm{ab}}$ & $6.52 \pm 0.22^{\mathrm{a}}$ & $6.38 \pm 0.04^{\mathrm{a}}$ & $5.84 \pm 0.07^{\mathrm{c}}$ & $5.86 \pm 0.15^{c}$ & $5.95 \pm 0.37^{\mathrm{bc}}$ & $6.25 \pm 0.25^{\mathrm{ab}}$ \\
\hline & Carnitine & $6.60 \pm 0.11^{\mathrm{ab}}$ & $6.51 \pm 0.13^{\mathrm{ab}}$ & $6.74 \pm 0.11^{\mathrm{a}}$ & $6.66 \pm 0.09^{\mathrm{ab}}$ & $5.64 \pm 0.14^{\mathrm{d}}$ & $5.67 \pm 0.10^{\mathrm{d}}$ & $6.01 \pm 0.40^{c}$ & $6.32 \pm 0.36^{\text {bc }}$ \\
\hline & Choline and derivatives & $7.04 \pm 0.15^{\mathrm{ab}}$ & $7.05 \pm 0.15^{\mathrm{ab}}$ & $7.26 \pm 0.17^{a}$ & $7.17 \pm 0.08^{\mathrm{ab}}$ & $6.24 \pm 0.09^{\mathrm{d}}$ & $6.32 \pm 0.06^{\mathrm{d}}$ & $6.65 \pm 0.32^{\mathrm{c}}$ & $6.91 \pm 0.33^{\text {bc }}$ \\
\hline & Glycerophosphocholine & $6.64 \pm 0.20^{\mathrm{a}}$ & $6.59 \pm 0.24^{\mathrm{a}}$ & $6.45 \pm 0.46^{\mathrm{ab}}$ & $5.65 \pm 1.45^{\mathrm{b}}$ & $6.42 \pm 0.10^{\mathrm{ab}}$ & $6.47 \pm 0.11^{\mathrm{ab}}$ & $6.70 \pm 0.23^{\mathrm{a}}$ & $6.87 \pm 0.11^{\mathrm{a}}$ \\
\hline \multirow{13}{*}{$\begin{array}{l}\text { Organic acids } \\
\text { and } \\
\text { derivatives }\end{array}$} & Acetate & $6.78 \pm 0.03^{\mathrm{a}}$ & $6.59 \pm 0.12^{\mathrm{ab}}$ & $6.87 \pm 0.11^{\mathrm{a}}$ & $6.75 \pm 0.01^{\mathrm{a}}$ & $5.99 \pm 0.13^{c}$ & $5.97 \pm 0.18^{\mathrm{c}}$ & $6.11 \pm 0.37^{\mathrm{c}}$ & $6.40 \pm 0.25^{b}$ \\
\hline & Acetoacetate & $6.45 \pm 0.07^{\mathrm{ab}}$ & $6.22 \pm 0.25^{\mathrm{ab}}$ & $6.52 \pm 0.22^{\mathrm{a}}$ & $6.37 \pm 0.08^{\mathrm{ab}}$ & $5.60 \pm 0.22^{\mathrm{d}}$ & $5.55 \pm 0.14^{\mathrm{d}}$ & $5.73 \pm 0.45^{\mathrm{cd}}$ & $6.08 \pm 0.40^{\mathrm{bc}}$ \\
\hline & Benzoate & $8.11 \pm 0.05^{\mathrm{a}}$ & $7.93 \pm 0.10^{\mathrm{ab}}$ & $8.21 \pm 0.04^{\mathrm{a}}$ & $8.13 \pm 0.04^{\mathrm{a}}$ & $7.27 \pm 0.11^{\text {de }}$ & $7.19 \pm 0.24^{\mathrm{e}}$ & $7.50 \pm 0.36^{\mathrm{cd}}$ & $7.70 \pm 0.25^{\mathrm{bc}}$ \\
\hline & Butyrate & $6.51 \pm 0.13^{\mathrm{a}}$ & $5.86 \pm 0.60^{\mathrm{ab}}$ & $6.35 \pm 0.64^{\mathrm{a}}$ & $6.11 \pm 0.31^{\mathrm{ab}}$ & $5.06 \pm 0.39^{c}$ & $4.94 \pm 0.64^{\mathrm{c}}$ & $5.45 \pm 0.69^{\mathrm{bc}}$ & $5.91 \pm 0.44^{\mathrm{ab}}$ \\
\hline & Citrate & $7.59 \pm 0.04^{\mathrm{a}}$ & $7.41 \pm 0.14^{\mathrm{ab}}$ & $7.69 \pm 0.11^{\mathrm{a}}$ & $7.58 \pm 0.05^{\mathrm{a}}$ & $6.70 \pm 0.13^{\mathrm{d}}$ & $6.69 \pm 0.17^{\mathrm{d}}$ & $6.98 \pm 0.37^{\mathrm{cd}}$ & $7.19 \pm 0.33^{\text {bc }}$ \\
\hline & Fumarate & $7.00 \pm 0.05^{\mathrm{a}}$ & $6.66 \pm 0.17^{\mathrm{ab}}$ & $6.85 \pm 0.14^{\mathrm{ab}}$ & $6.74 \pm 0.10^{\mathrm{ab}}$ & $6.54 \pm 0.10^{\mathrm{ab}}$ & $6.44 \pm 0.10^{\mathrm{ab}}$ & $6.29 \pm 0.83^{b}$ & $6.46 \pm 0.63^{\mathrm{ab}}$ \\
\hline & Hippurate & $8.28 \pm 0.02^{\mathrm{a}}$ & $8.08 \pm 0.11^{\mathrm{ab}}$ & $8.33 \pm 0.05^{\mathrm{a}}$ & $8.27 \pm 0.06^{\mathrm{a}}$ & $7.47 \pm 0.12^{\mathrm{cd}}$ & $7.31 \pm 0.28^{\mathrm{d}}$ & $7.60 \pm 0.48^{\mathrm{cd}}$ & $7.79 \pm 0.42^{\mathrm{bc}}$ \\
\hline & $\beta$-hydroxybutyrate & $6.98 \pm 0.08^{\mathrm{a}}$ & $6.77 \pm 0.20^{\mathrm{ab}}$ & $7.09 \pm 0.23^{\mathrm{a}}$ & $6.93 \pm 0.03^{\mathrm{a}}$ & $6.08 \pm 0.11^{\mathrm{d}}$ & $6.10 \pm 0.24^{\mathrm{d}}$ & $6.19 \pm 0.48^{\mathrm{cd}}$ & $6.54 \pm 0.33^{\text {bc }}$ \\
\hline & Isobutyrate & $6.56 \pm 0.13^{\mathrm{a}}$ & $6.09 \pm 0.48^{\mathrm{ab}}$ & $6.51 \pm 0.45^{\mathrm{a}}$ & $6.30 \pm 0.21^{\mathrm{a}}$ & $5.23 \pm 0.26^{c}$ & $5.14 \pm 0.61^{\mathrm{c}}$ & $5.63 \pm 0.43^{\mathrm{bc}}$ & $5.95 \pm 0.50^{\mathrm{ab}}$ \\
\hline & Lactate & $6.60 \pm 0.16^{\mathrm{a}}$ & $6.11 \pm 0.67^{\mathrm{a}}$ & $6.36 \pm 0.59^{\mathrm{a}}$ & $6.08 \pm 0.43^{\mathrm{a}}$ & $6.04 \pm 0.15^{\mathrm{a}}$ & $6.24 \pm 0.19^{a}$ & $6.34 \pm 0.29^{a}$ & $6.59 \pm 0.25^{\mathrm{a}}$ \\
\hline & Orotate & $8.29 \pm 0.06^{\mathrm{ab}}$ & $8.28 \pm 0.09^{\mathrm{ab}}$ & $8.27 \pm 0.03^{\mathrm{ab}}$ & $8.23 \pm 0.05^{\mathrm{b}}$ & $8.38 \pm 0.10^{\mathrm{a}}$ & $8.30 \pm 0.07^{\mathrm{ab}}$ & $8.29 \pm 0.09^{\mathrm{ab}}$ & $8.25 \pm 0.08^{b}$ \\
\hline & Succinate & $6.95 \pm 0.04^{\mathrm{ab}}$ & $6.78 \pm 0.15^{\mathrm{bc}}$ & $7.08 \pm 0.18^{\mathrm{a}}$ & $6.95 \pm 0.02^{\mathrm{ab}}$ & $6.10 \pm 0.12^{\mathrm{e}}$ & $6.15 \pm 0.15^{\mathrm{e}}$ & $6.38 \pm 0.33^{\text {de }}$ & $6.60 \pm 0.29^{\mathrm{cd}}$ \\
\hline & Valerate and derivatives & $7.30 \pm 0.11^{\mathrm{a}}$ & $6.80 \pm 0.46^{\mathrm{abc}}$ & $7.27 \pm 0.37 \mathrm{ab}$ & $7.04 \pm 0.19^{\mathrm{ab}}$ & $5.99 \pm 0.21^{\mathrm{d}}$ & $5.91 \pm 0.48^{\mathrm{d}}$ & $6.33 \pm 0.46^{\mathrm{cd}}$ & $6.69 \pm 0.50^{\mathrm{bc}}$ \\
\hline
\end{tabular}

Note: Metabolite contents are expressed as $\log 10$ [peak area of respective compound in arbitrary unit].

Values are the average from four individual cow samples \pm SD corresponding to the respective day after parturition, farm, and sampling period.

Superscript letters $(\mathrm{a}-\mathrm{e})$ indicate significant difference $(\mathrm{P} \leq 0.05)$ among sample means within the same row.

* Period I corresponds to sampling during October 2018.

** Period II corresponds to sampling during January 2019. 
An overall PLS-DA score plot was constructed based on the first two components with $R^{2}=0.703$ and $Q^{2}=0.628$ (Figure 3A). A good distinction was observed between colostrum samples collected on day-1 (marked in red) and day-3 (marked in green) postpartum along with component 1 (76.42\% variance). VIP scores indicated that high relative abundances of threonine, isoleucine, tyrosine, ethanol, iso-butyrate, valerate, butyrate, valine, and histidine were accountable for the discrimination of colostrum collected on day-1 postpartum. On the other hand, high relative abundances of lactose, glucose, ascorbate, glycerol-phosphocholine, galactose, and orotate were accountable for the discrimination of colostrum collected on day-3 postpartum. The integrated intensities of these potential biomarker metabolites, expressed in $\log _{10}$ transformed values (arbitrary unit), were compared through a box-whicker plot summary (Figure 3C).

For evaluating the main effect of farm and sampling month, two separated PLS-DA score plots were constructed for distinguishing the ${ }^{1} \mathrm{H}-\mathrm{NMR}$ derived metabolomic profiles within the group of day-1 postpartum samples $(n=16)$ with $R^{2}=0.451$ and $Q^{2}=0.124$ (Figure $4 \mathrm{~A}$ ) and day-3 postpartum samples $(n=16)$ with $R^{2}=0.633$ and $Q^{2}=0.295$ (Figure 4B). Within the group of day-1 postpartum samples, the influence of sampling month on non-volatile metabolite profiles of colostrum was notably observed in samples collected within the same

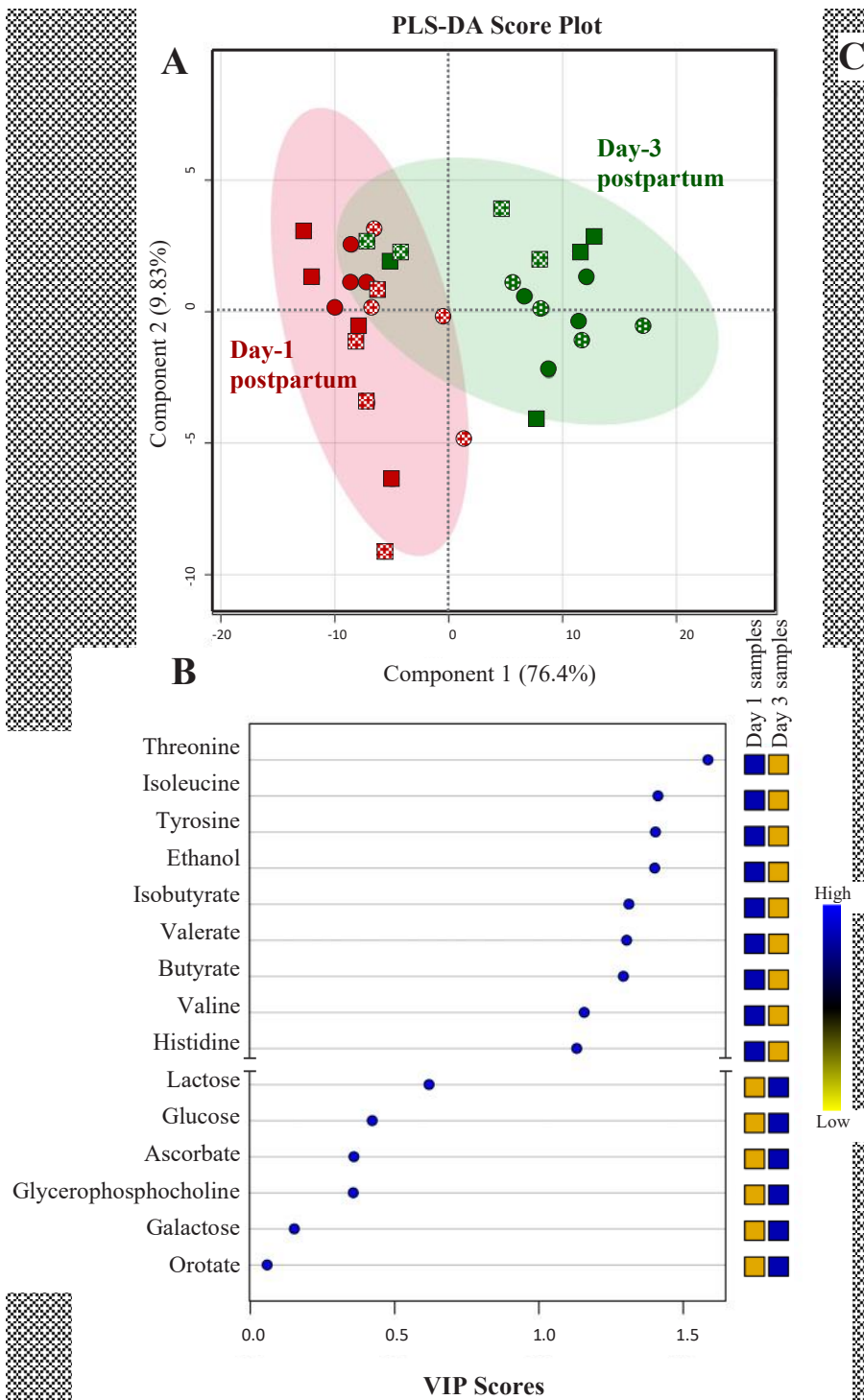

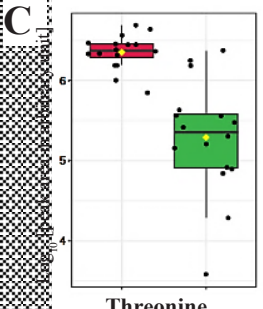

Threonine

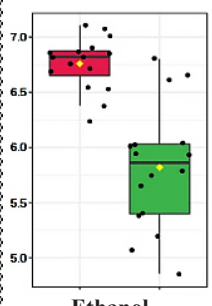

Ethanol

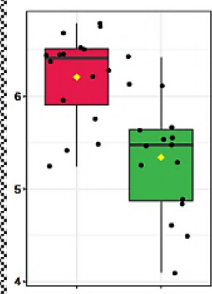

Butyrate

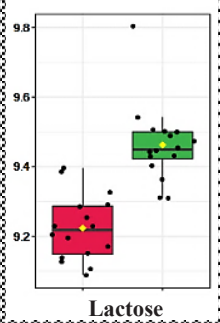

Lactose

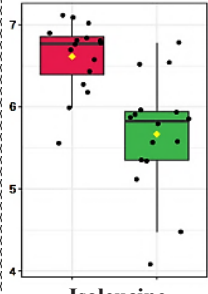

Isoleucine

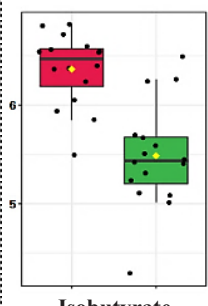

Isobutyrate
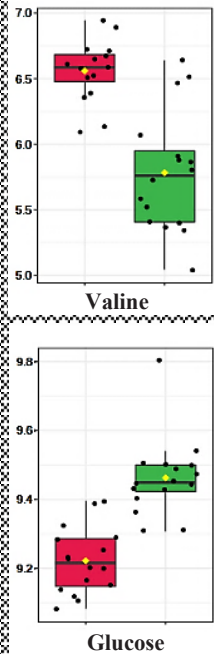

Glucose
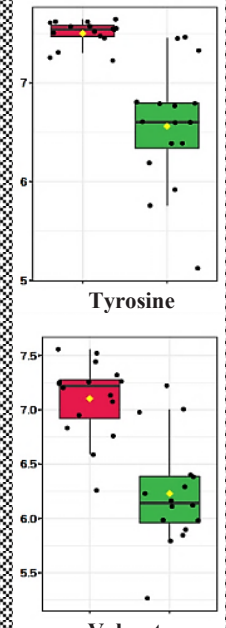

Valerate

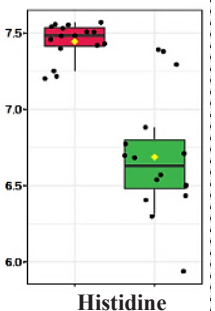

Histidine

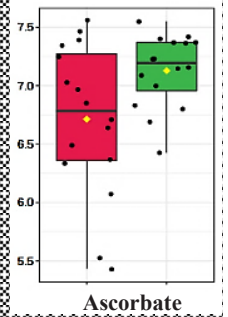

Figure 3. PLS-DA score plot (panel A) and VIP scores (panel B) for an overall comparison of non-volatile metabolite profiles of colostrum samples collected from individual cows in farm A during period I (October 2018; $\bullet$ ), farm A during period II (January 2019; ®), farm B during period I (October 2018; $\square$ ), and farm B during period II (January 2019; ॠ). Box-whisker plots of comparative quantification of potential biomarker metabolites ( $\log$ [peak area of respective compound in arbitrary unit]) responsible for discrimination between colostrum samples collected on day-1 ( $\square$ ) and day-3 ( $\square$ ) postpartum (panel C). The lower and upper edge of the box denote $25^{\text {th }}$ and $75^{\text {th }}$ percentile of observation, respectively; the bold line within box denotes median value; the yellow-spot within box denotes average value; whiskers denote $5^{\text {th }}$ and $95^{\text {th }}$ percentiles. For interpretation of the references to color in this figure, the reader is referred to the web version of this article and the relative quantification of metabolites in supplementary Table S1. 
farm. This effect remained consistent for the two farms. It was remarkable that the difference between farms was less pronounced in samples collected in period I. In other words, a distinct pattern between farms was clearly noticed among samples collected in period II. Within the group of day-3 postpartum samples, the influence of farm and sampling month on non-volatile metabolite profiles of colostrum was unlikely pronounced in samples of farm A and those collected in period I of farm B. It was remarkable that the non-volatile metabolite profile of samples collected in period II of farm B were completely distinguished from other groups.

\section{DISCUSSION}

It has been documented that the $\mathrm{pH}$ of bovine colostrum is low initially (6.0-6.3) and increases progressively to the level of mature milk (6.6-6.8) (McGrath et
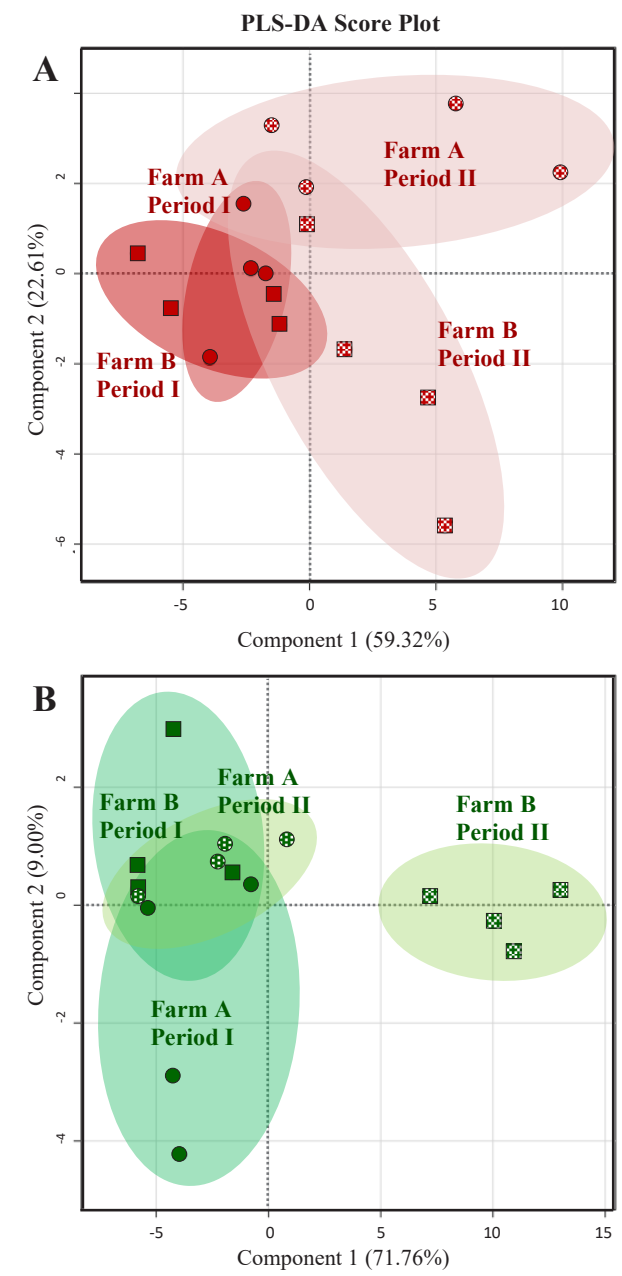

Figure 4. PLS-DA score plots, for comparison among samples collected on day-1 (panel A) and day-3 (panel B) postpartum, derived from of non-volatile metabolite profiles of colostrum samples collected from individual cows in farm A during period I (October 2018; @), farm A during period II (January 2019; $\otimes$ ), farm B during period I (October 2018; $\mathbf{\square})$, and farm B during period II (January 2019; ॠ). For interpretation of the references to color in this figure, the reader is referred to the web version of this article. al., 2016; Tsioulpas et al., 2007). The range of colostrum $\mathrm{pH}$ and titratable acidity observed in this study and their substantial changes from day- 1 to day-3 postpartum (Table 1) were in agreement with the literature (McGrath et al., 2016; Tsioulpas et al., 2007). Regarding rheological property, our results demonstrated a significant decrease in the viscosity of colostrum with time postpartum (Table 1). A positive correlation between the decrease in colostrum viscosity and the reduction of proteins and immunoglobulin (IgG) contents with time postpartum has been reported (Ceniti et al., 2019; McGrath et al., 2016). The CIE $L^{*} a^{*} b^{*}$ color coordinate results demonstrated that the yellowness $\left(+b^{*}\right)$ of colostrum samples significantly decreased over the first 3 days postpartum (Table 1). This finding corresponds with the work of Madsen et al. (2004) and Calderón et al. (2007), who described that the color of colostrum became less yellow with time after calving due to the decline in carotenoids, lutein, and $\beta$-carotene contents. Interestingly, we only found a significant impact of the farm on the $\mathrm{pH}$, titratable acidity, viscosity, and color range of colostrum samples collected on day-3 postpartum (Table 1). Variations in these physical properties could be attributed to various factors in the dairy production system (Calderón et al., 2007; Dunn et al., 2017; Madsen et al., 2004; McGrath et al., 2016). Practically, these physical parameters are widely used to assess the quality of colostrum, especially in relation to the immunoglobulins concentration (Dunn et al., 2017; Madsen et al., 2004; McGrath et al., 2016).

The concentration of fat, proteins, and lactose of colostrum samples in this study was compliant with the ranges reported in kinds of literature (Godhia \& Patel, 2013; McGrath et al., 2016; Puppel et al., 2019). It is well documented that colostrum contains a significantly higher content of proteins (ca. 14.9\%), especially IgG, IgM, and IgA, than mature milk (ca. 3.2\%) (McGrath et al., 2016). The higher level of this passive immunity in the colostrum allows the calves to defend against diseases and harsh environments. However, the concentration of these immunoglobulins declines rapidly after parturition resulting in a sharp decrease in total protein content (Madsen et al., 2004; Puppel et al., 2019). Our results confirm the significantly higher protein content in samples collected on day-1 compared to day-3 postpartum. In contrast, a significant increase in lactose concentration was noticed in samples collected on day-3 postpartum (Table 1). This result is in agreement with several authors who reported a low level of lactose in early colostrum after parturition (ca. $2.5 \%$ ) followed by a steady increase to the level of mature milk (ca. 4.5\%) (Madsen et al., 2004; Tsioulpas et al., 2007). Since lactose is an osmoregulatory responsible for the influx of water into milk, a low level of this sugar results in less water excretion with less water and therefore generates low milk yield with extremely viscous characteristics (McGrath et al., 2016). Accordingly, physical properties, i.e., specific gravity, density, and viscosity of colostrum, can be affected (Ceniti et al., 2019; McGrath et al., 2016). The significant rise in lactose and decline in protein content during the first 3 days postpartum found here have well corresponded with the decrease in viscos- 
ity of samples discussed earlier. Similar to the physical property results, a significant impact of the farm on the major chemical constituents of colostrum was only observed within the group of samples collected on day-3 postpartum (Table 1). Besides the gross composition, colostrum also contains various small molecular weight metabolites, which are also important to stimulate the growth and promote the health of newborns.

Recently, ${ }^{1} \mathrm{H}-\mathrm{NMR}$ has been broadly applied in metabolite profiling of colostrum, milk, and dairy products (Scano et al., 2019). The list of metabolites identified in this study (45 compounds) is comparable with those detected in bovine, sow, and human colostrum in other publications using a ${ }^{1} \mathrm{H}-\mathrm{NMR}$ platform (500-600 MHz.) (Luise et al., 2020; Picone et al., 2018; Wu et al., 2016; Zhao et al., 2018). As expected, variability in the detected metabolites was however observed among studies. The reasons may be due to the impact of animals and environmental factors on the nature of samples, as well as NMR sample preparation methods, that can induce significant alterations in the metabolome of colostrum (Denholm et al., 2018; Puppel et al., 2019). HCA and PLSDA patterns confirm a complete distinction between metabolite profiles of the samples collected on day-1 and day-3 postpartum (Figure 2-3). It should be mentioned that most of the metabolites were present in high relative abundance in samples collected on day-1 postpartum (Figure 2; Table 3). Of these, five amino acids, i.e., threonine, isoleucine, tyrosine, valine, and histidine, were identified as biomarker metabolites accountable for the discrimination with the highest VIP scores and $\mathrm{p}<0.05$ (Figure 3B). The high abundance of these metabolites is in agreement with previous works on the dynamics of amino acid profile in colostrum of dairy cattle (Csapó et al., 2012; Kráčmar et al., 2007; Li et al., 2020), goat (Kráčmar et al., 1999), camel (Kamal et al., 2007), as well as human (Zhang et al., 2013). From a nutritional viewpoint, threonine can promote the growth and development of infants and enhance immunity and the development of lymphocytes (Li et al., 2020). Isoleucine and valine are branched-chain essential amino acids that participate in up-regulating innate and adaptive immune responses and enhance muscle protein synthesis (Zhang et al., 2017). Tyrosine is an essential precursor in the biosynthesis of various neurotransmitters. Histidine participates in the development of the infant nervous system (Li et al., 2020). The presence of amino acids in a high relative abundance could be associated with the high total protein content in day-1 samples as discussed earlier. Besides amino acids, iso-butyrate, valerate, and butyrate were also identified as biomarker metabolites accountable for the discrimination between samples collected on day-1 and day-3 postpartum (VIP > 1.0; $\mathrm{p}<0.05)$ (Figure 3B). These volatile fatty acids are derived from rumen fermentation and directly affect milk fat biosynthesis capacities in the udders ( $\mathrm{O}^{\prime}$ Callaghan et al., 2020). The function of butyrate to enhance calf growth and intestinal development has been well acknowledged (Górka et al., 2018; O'Callaghan et al., 2020). Moreover, we found a positive correlation between high proteins in day- 1 colostrum with choline, $N$-acetyl glucosamine, $\mathrm{N}$-acetyl galactosamine, and creatinine (Figure 2; Table
3) as also reported in mature milk by Sundekilde et al. (2014). Nevertheless, inconsistent changes in isoleucine, histidine, and butyrate in bovine colostrum during the first week postpartum were reported in several works (Cui et al., 2015; Meklati et al., 2019; O'Callaghan et al., 2020; Zándoki et al., 2006). This contradiction may be attributed to variations among animals, experimental environments, and detection methods. Besides the metabolites rich in the first day colostrum, lactose, glucose, galactose, ascorbate, glycerol-phosphocholine, orotate, and lactate were present in significantly or likely higher concentration in samples collected on day-3 postpartum (Figure 2-3; Table 3). The high level of sugars noticed here corresponds well with the high content of lactose discussed earlier in the gross composition analysis. Glycerophosphocholine is formed during the breakdown of phosphatidylcholine to attain fatty acids for the synthesis of milk triglycerides and phospholipids (Klein et al., 2012). Orotate (vitamin B-13) is a compound derived from pyrimidine metabolism (Robinson, 1980), which greatly contributes to the somatosensory flavor profiles of liquid milk products (Potts \& Peterson, 2019). Our results successfully revealed time-dependent changes in the metabolome of bovine colostrum, with differential biomarker metabolites, during the first three days after calving. Moreover, it should be mentioned that the mammary gland secretes diverse components via (i) direct transfer of components from the maternal bloodstream into mammary secretions in the first colostrum after calving and thereafter (ii) through the local synthesis in milk secreting cells with the increased time postpartum (McGrath et al., 2016). Therefore, the trends increase or decrease of each metabolite measured could be suggested whether the compound is secreted by the milk epithelial secreting cells or either enter to the lumen by transportation from the bloodstream or possibly generated by other non-mammary gland cells. For example, the abundance of free amino acid levels in the fisrt day colostrum could be attributed to the facilitated diffusion through epithelial cells via amino acid transporters together with the higher levels of proteases, which generate free amino acids and peptides from milk protein hydrolysis, in early colostrum compared to transient milk (Li et al., 2020; McGrath et al., 2016).

The main effects of farm and sampling month on non-volatile metabolite profiles of colostrum collected at the same time postpartum are presented in Figure 4. Although no significant change in the physical property, with an exception for the yellowness $\left({ }^{*} b\right)$, and major chemical constituents was observed among samples collected on day-1 postpartum (Table 1), variations in their metabolite profiles influenced by farm origin and sampling month were remarkably revealed by PLSDA (Figure 4A). On day-3 postpartum, the metabolite profiles of samples from farm B during sampling period II were completely distinguished from other groups (Figure 4B). The PLS-DA pattern highlighted their exclusive non-volatile metabolite profiles further than their physical properties and major chemical constituents (Table 1). Future research focusing on the effect of these parameters on colostrum metabolome, especially in tropical dairy farming environments, could be of 
great interest. Although variations in milk composition associated with the origin of the farm (many factors involved) and sampling period (milking time, seasons, and climate) have been well acknowledged (Promket et al., 2020; Puppel et al., 2019), available data of these effects on the overall metabolite profile or molecular fingerprint of colostrum is rather limited. This information could help to suggest directions for improving dairy management to achieve the desired colostrum quality.

Our findings support the effectiveness of using non-targeted ${ }^{1} \mathrm{H}$-NMR metabolomics to investigate the influence of time after parturition, farm origin, as well as sample collecting month on the molecular fingerprints of bovine colostrum. In addition to basic nutritive compounds (amino acids and sugars), several bioactive metabolites such as ascorbate, creatine, carnitine, choline, acetylcarnitine, $\mathrm{N}$-acetylglucosamine, ornithine, orotate, and UPD-glucose could also be elucidated (Figure 2; Table 3). This result suggests that colostrum metabolite profiles should be essentially deliberated from a nutritional point of view. Since bovine colostrum has been recently introduced as feed supplements and functional ingredients in food and nutraceuticals industries, the identified differential metabolites may serve as potential biomarkers for a quality control purpose, besides the amount of gross composition and antibody, in the future.

\section{CONCLUSION}

In the present study, non-volatile polar metabolite profiles of bovine colostrum collected during the first three days after parturition were characterized and compared using a non-targeted ${ }^{1} \mathrm{H}-\mathrm{NMR}$ metabolomics approach. A total of 45 metabolites were identified. The post-parturition time provided a significant impact on the metabolome of colostrum. Dynamic changes in certain nutrients and bioactive metabolites were significantly observed between colostrum collected on the first and third day postpartum. In addition, the influence of farm origin and sampling month on the metabolome of colostrum could be revealed by the chemometric analysis.

\section{CONFLICT OF INTEREST}

The authors declare no conflict of interest with respect to this work.

\section{ACKNOWLEDGEMENT}

This study was conducted under the New Scholar Ratchadaphiseksomphot Endowment Fund (RGN-2559013-06-23) from Chulalongkorn University and partially supported by open innovation grant (P12-BE-61-01-004) from National Innovation Association, Ministry of Science and Technology of Thailand. Technical assistances provided by Aunchisa Kuntaveesuk and Marisa Kongboonkird were highly appreciated.

\section{REFERENCES}

Afshari, R., C. J. Pillidge, D. A. Dias, A. M. Osborn, \& H. Gill. 2020. Cheesomics: The future pathway to understanding cheese flavour and quality. Crit. Rev. Food Sci. Nutr. 60:3347. https://doi.org/10.1080/10408398.2018.1512471

Calderón, F., B. Chauveau-Duriot, B. Martin, B. Graulet, M. Doreau, \& P. Nozière. 2007. Variations in carotenoids, vitamins $\mathrm{A}$ and $\mathrm{E}$, and color in cow's plasma and milk during late pregnancy and the first three months of lactation. J. Dairy Sci. 90:2335-2346. https://doi.org/10.3168/ jds.2006-630

Ceniti, C., F. Froiio, D. Britti, D. Paolino, \& N. Costanzo. 2019. Rheological characteristics of bovine colostrum and their correlation with immunoglobulin G. Int. J. Dairy Technol. 72:345-349. https://doi.org/10.1111/1471-0307.12593

Csapó, J., B. Béri, A. Süli, \& E. Varga-Visi. 2012. Colostrum and milk of different cattle breeds as amino acid source. Acta Agric. Slov. 100:327-331.

Cui, J., D. Zhu, M. Su, D. Tan, X. Zhang, M. Jia, \& G. Chen. 2019. The combined use of $1 \mathrm{H}$ and $2 \mathrm{D}$ NMR-based metabolomics and chemometrics for non-targeted screening of biomarkers and identification of reconstituted milk. J. Sci. Food Agric. 99:6455-6461. https://doi.org/10.1002/jsfa.9924

Cui, N., P. C. Wen, Q. Liang, H. N. Liu, W. B. Zhang, P. J. Wang, H. Y. Guo, \& F. Z. Ren. 2015. Chemical composition of yak colostrum and transient milk. J. Anim. Physiol. Anim. Nutr. 99:825-833. https://doi.org/10.1111/jpn.12282

Denholm, K. S., S. McDougall, G. Chambers, \& W. Clough. 2018. Factors associated with colostrum quality in individual cows from dairy herds in the Waikato region of New Zealand. N. Z. Vet. J. 66:115-120. https://doi.org/10.1080/00 480169.2017.1418684

Dunn, A., A. Ashfield, B. Earley, M. Welsh, A. Gordon, \& S. J. Morrison. 2017. Evaluation of factors associated with immunoglobulin $G$, fat, protein, and lactose concentrations in bovine colostrum and colostrum management practices in grassland-based dairy systems in Northern Ireland. J. Dairy Sci. 100:2068-2079. https://doi.org/10.3168/ jds.2016-11724

Foroutan, A., A. C. Guo, R. Vazquez-Fresno, M. Lipfert, L. Zhang, J. Zheng, H. Badran, Z. Budinski, R. Mandal, B. N. Ametaj, \& D. S. Wishart. 2019. Chemical composition of commercial cow's milk. J. Agric. Food Chem. 67:48974914. https://doi.org/10.1021/acs.jafc.9b00204

Godhia, M. L. \& N. Patel. 2013. Colostrum - Its composition, benefits as a nutraceutical: A review. Curr. Res. Nutr. Food Sci. 1:37-47. https://doi.org/10.12944/CRNFSJ.1.1.04

Goldansaz, S. A., A. C. Guo, T. Sajed, M. A. Steele, G. S. Plastow, \& D. S. Wishart. 2017. Livestock metabolomics and the livestock metabolome: A systematic review. PLoS ONE. 12: e0177675. https://doi.org/10.1371/journal. pone.0177675

Górka, P., Z. M. Kowalski, R. Zabielski, \& P. Guilloteau. 2018. Invited review: Use of butyrate to promote gastrointestinal tract development in calves. J. Dairy Sci. 101:4785-4800. https://doi.org/10.3168/jds.2017-14086

Gross, J. J., E. C. Kessler, \& R. M. Bruckmaier. 2014. Colour measurement of colostrum for estimation of colostral IgG and colostrum composition in dairy cows. J. Dairy Res. 81:440-444. https://doi.org/10.1017/S0022029914000466

ISO/IDF. 2012. ISO/TS11869 - IDF/RM150:2012 - Fermented milks - determination of titratable acidity - potentiometric method. International Organization for Standardization, Geneva.

Kamal, A. M., O. A. Salama, \& K. M. El-Saied. 2007. Changes in amino acids profile of camel milk protein during the early lactation. Int. J. Dairy Sci. 2:226-234. https://doi. org/10.3923/ijds.2007.226.234 
Klein, M. S., N. Buttchereit, S. P. Miemczyk, A.-K. Immervoll, C. Louis, S. Wiedemann, W. Junge, G. Thaller, P. J. Oefner, \& W. Gronwald. 2012. NMR metabolomic analysis of dairy cows reveals milk glycerophosphocholine to phosphocholine ratio as prognostic biomarker for risk of ketosis. J. Proteome Res. 11:1373-1381. https://doi. org/10.1021/pr201017n

Kráčmar, S., S. Gajdůšek, P. Jelínek, L. Zeman, V. Kozel, M. Kozlová, \& E. Kráčmarová. 1999. Changes in amino acid composition of goat's colostrum during the first 72 hours after birth. Czech J. Anim. Sci. 44:541-545.

Kráčmar, S., F. Buňka, I. Hoza, L. Čechová, \& P. Valášek. 2007. Changes in amino acids composition of cows colostrum (during first 72 hours after parturition). Acta Univ. Agric. Silvic. Mendel. Brun. 55:81-94. https://doi.org/10.11118/ actaun200755010081

Li, M., W. Li, F. Kong, S. Kang, X. Liang, H. Han, J. Wu, Y. Zheng, Q. Li, X. Yue, \& M. Yang. 2020. Metabolomics methods to analyze full spectrum of amino acids in different domains of bovine colostrum and mature milk. Eur. Food Res. Technol. 246:213-224. https://doi.org/10.1007/ s00217-019-03385-y

Luangwilai, M., K. Duangmal, N. Chantaprasarn \& S. Settachaimongkon. 2021. Comparative metabolite profiling of raw milk from subclinical and clinical mastitis cows using ${ }^{1} \mathrm{H}-\mathrm{NMR}$ combined with chemometric analysis. Int. J. Food Sci. Tech. 56: 493-503. https://doi.org/10.1111/ ijfs.14665

Luise, D., G. Picone, A. Balzani, F. Capozzi, M. Bertocchi, C. Salvarani, P. Bosi, S. Edwards, \& P. Trevisi. 2020. Investigation of the defatted colostrum1H-NMR metabolomics profile of gilts and multiparous sows and its relationship with litter performance. Animals. 10:154. https:// doi.org/10.3390/ani10010154

Madsen, B. D., M. D. Rasmussen, M. O. Nielsen, L. Wiking, \& L. B. Larsen. 2004. Physical properties of mammary secretions in relation to chemical changes during transition from colostrum to milk. J. Dairy Res. 71:263-272. https:// doi.org/10.1017/S0022029904000263

McGrath, B. A., P. F. Fox, P. L. H. McSweeney, \& A. L. Kelly. 2016. Composition and properties of bovine colostrum: a review. Dairy Sci. Technol. 96:133-158. https://doi. org/10.1007/s13594-015-0258-x

Meklati, F. R., A. Meribai, N. Yezli, \& T. Benabdelaziz. 2019. Colostrum and milk fatty acids profiles from imported prim'Holstein cows. Pertanika J. Trop. Agric. Sci. 42:595-607.

O'Callaghan, T. F., M. O'Donovan, J. P. Murphy, K. Sugrue, D. Mannion, W. P. McCarthy, M. Timlin, K. N. Kilcawley, R. M. Hickey, \& J. T. Tobin. 2020. Evolution of the bovine milk fatty acid profile - From colostrum to milk five days post parturition. Int. Dairy J. 104:104655. https://doi. org/10.1016/j.idairyj.2020.104655

Penchev Georgiev, I. 2008. Differences in chemical composition between cow colostrum and milk. Bulg. J. Vet. Med. 1:3-12.

Picone, G., M. Zappaterra, D. Luise, A. Trimigno, F. Capozzi, V. Motta, R. Davoli, L. Nanni Costa, P. Bosi, \& P. Trevisi. 2018. Metabolomics characterization of colostrum in three sow breeds and its influences on piglets' survival and litter growth rates. J. Anim. Sci. Biotechnol. 9:23. https://doi. org/10.1186/s40104-018-0237-1

Potts, D. M. \& D. G. Peterson. 2019. Identification of small molecule flavor compounds that contribute to the somatosensory attributes of bovine milk products. Food Chem. 294:27-34. https://doi.org/10.1016/j.foodchem.2019.05.010

Promket, D., W. Kenchaiwong, \& K. Ruangwittayanusorn. 2020. Effects of climate change on milk yield and milk composition in thai crossbred holstein cows.
International Journal of GEOMATE 18:108-113. https://doi. org $/ 10.21660 / 2020.67 .5807$

Puppel, K., M. Gołębiewski, G. Grodkowski, J. Slósarz, M. Kunowska-Slósarz, P. Solarczyk, M. Łukasiewicz, M. Balcerak, \& T. Przysucha. 2019. Composition and factors affecting quality of bovine colostrum: A review. Animals. 9:1070. https://doi.org/10.3390/ani9121070

Qi, Y., X. Zhao, D. Huang, X. Pan, Y. Yang, H. Zhao, H. Hu, \& G. Cheng. 2018. Exploration of the Relationship between Intestinal Colostrum or Milk, and Serum Metabolites in Neonatal Calves by Metabolomics Analysis. J. Agric. Food Chem. 66:7200-7208. https://doi.org/10.1021/acs. jafc. $8 \mathrm{~b} 01621$

Robinson, J. L. 1980. Bovine milk orotic acid: Variability and significance for human nutrition. J. Dairy Sci. 63:865-871. https://doi.org/10.3168/jds.S0022-0302(80)83017-7

Scano, P., E. Cusano, P. Caboni, \& R. Consonni. 2019. NMR metabolite profiles of dairy: A review. Int. Dairy J. 90:5667. https://doi.org/10.1016/j.idairyj.2018.11.004

Settachaimongkon, S., M. J. R. Nout, E. C. Antunes Fernandes, K. A. Hettinga, J. M. Vervoort, T. C. M. van Hooijdonk, M. H. Zwietering, E. J. Smid, \& H. J. F. van Valenberg. 2014. Influence of different proteolytic strains of Streptococcus thermophilus in co-culture with Lactobacillus delbrueckii subsp. bulgaricus on the metabolite profile of set-yoghurt. Int. J. Food Microbiol. 177: 29-36. https://doi.org/10.1016/j. ijfoodmicro.2014.02.008

Settachaimongkon, S., H. J. F. van Valenberg, \& E. J. Smid. 2017. Metabolomics as an emerging strategy for the investigation of yogurt components, in: N. P. Shah (Ed.), Yogurt in Health and Disease Prevention. Academic Press, London, pp. 427-449. https://doi.org/10.1016/ B978-0-12-805134-4.00025-0

Sundekilde, U. K., F. Gustavsson, N. A. Poulsen, M. Glantz, M. Paulsson, L. B. Larsen, \& H.C. Bertram. 2014. Association between the bovine milk metabolome and rennet-induced coagulation properties of milk. J. Dairy Sci. 97: 6076-6084. https://doi.org/10.3168/jds.2014-8304

Tsioulpas, A., A. S. Grandison, \& M. J. Lewis. 2007. Changes in physical properties of bovine milk from the colostrum period to early lactation. J. Dairy Sci. 90:5012-5017. https:// doi.org/10.3168/jds.2007-0192

Wu, J., M. Domellöf, A. M. Zivkovic, G. Larsson, A. Öhman, \& M. L. Nording. 2016. NMR-based metabolite profiling of human milk: A pilot study of methods for investigating compositional changes during lactation. Biochem. Biophys. Res. Commun. 469:626-632. https://doi.org/10.1016/j. bbrc.2015.11.114

Zándoki, R., J. Csapó, Z. Csapó-Kiss, I. Tábori, Z. Domokos, E. Szucs, \& J. Tozsér. 2006. Change of amino acid profile in Charolais cows' colostrum and transient milk during the first week post partum. Czech J. Anim. Sci. 51:375-382. https://doi.org/10.17221/3954-CJAS

Zhang, S., X. Zeng, M. Ren, X. Mau, \& S. Qiao. 2017. Novel metabolic and physiological functions of branched chain amino acids: A review. J. Anim. Sci. Biotechnol. 8:10. https://doi.org/10.1186/s40104-016-0139-z

Zhang, Z., A. S. Adelman, D. Rai, J. Boettcher, \& B. Lonnerdal. 2013. Amino acid profiles in term and preterm human milk through lactation: A systematic review. Nutrients. 5:48004821. https://doi.org/10.3390/nu5124800

Zhao, X. W., Y. X. Qi, D. W. Huang, X. C. Pan, G. L. Cheng, H. L. Zhao, \& Y. X. Yang. 2018. Changes in serum metabolites in response to ingested colostrum and milk in neonatal calves, measured by nuclear magnetic resonance-based metabolomics analysis. J. Dairy Sci. 101:7168-7181. https:// doi.org/10.3168/jds.2017-14287 